\title{
Variable Stars in the Globular Cluster M92
}

\author{
Grzegorz Kopacki \\ Wroclaw University Observatory, Kopernika 11, Poland
}

\begin{abstract}
Results of a search for variable stars in the central region of the Oosterhoff II type globular cluster M92 are presented.
\end{abstract}

Out of the 28 variable and suspected variable stars listed in the Catalogue of Variable Stars in Globular Clusters (Clement 1997), only two were not observed. Surprisingly, almost half of the observed suspected variables did not show any evidence of variability. Only one out of the 11 candidate RR Lyrae variables of Kadla et al. (1983) appeared to be variable. Moreover, variable v7, until now classified as an RR Lyrae star with a period of about $0.515 \mathrm{~d}$, turned out to be of the BL Herculis type, with the period approximately twice that long. In addition, six new variables were found in the very core of the cluster: four of the RR Lyrae type and the remaining two of the SX Phoenicis type. Altogether, light curves have been obtained for 20 variable stars. The total number of known RR Lyrae stars in M92 now equals 17; 11 of type RRab and the remaining 6, RRc.

$R R c$ variable $v 11$ shows changes in the light curve shape. Due to the aliasing problems, however, we can state only that this star is biperiodic. There is a possibility that v11 belongs to the new group of recently discovered doubleperiod RRc variables pulsating in non-radial mode (Olech et al. 1999).

Period-shift analysis for M92 and M2 has resulted in finding that, despite a significant difference in metal abundance between these two clusters $([\mathrm{Fe} / \mathrm{H}]=$ -2.24 for M92 and -1.62 for M2), there is no firm evidence for a shift in period.

Acknowledgments. This work was supported by Wroclaw University grant $2041 / \mathrm{W} / \mathrm{IA} / 2000$. GK gratefully acknowledges financial support through the IAU grant.

\section{References}

Clement, C. 1997, Preliminary Fourth Edition of the Catalogue on Variable Stars in Globular Clusters, electronic version.

Kadla Z.I., Yablokova N.V., Gerashchenko A.N., Spasova N. 1983, Peremennye Zvezdy, 21, 827

Olech A., Kalużny J., Thompson I.B., et al. 1999, AJ, 118, 442 